\title{
UNCOUNTABLE EQUILATERAL SETS IN BANACH SPACES OF THE FORM $C(K)$
}

\author{
PIOTR KOSZMIDER
}

\begin{abstract}
The paper is concerned with the problem whether a nonseparable Banach space must contain an uncountable set of vectors such that the distances between every two distinct vectors of the set are the same. Such sets are called equilateral. We show that Martin's axiom and the negation of the continuum hypothesis imply that every nonseparable Banach space of the form $C(K)$ has an uncountable equilateral set. We also show that one cannot obtain such a result without an additional set-theoretic assumption since we construct an example of nonseparable Banach space of the form $C(K)$ which has no uncountable equilateral set (or equivalently no uncountable $(1+\varepsilon)$ separated set in the unit sphere for any $\varepsilon>0$ ) making another consistent combinatorial assumption. The compact $K$ is a version of the split interval obtained from a sequence of functions which behave in an anti-Ramsey manner. It remains open if there is an absolute example of a nonseparable Banach space of the form different than $C(K)$ which has no uncountable equilateral set. It follows from the results of S. Mercourakis, G. Vassiliadis that our example has an equivalent renorming in which it has an uncountable equilateral set. It remains open if there are consistent examples which have no uncountable equilateral sets in any equivalent renorming but it follows from the results of $\mathrm{S}$. Todorcevic that it is consistent that every nonseparable Banach space has an equivalent renorming in which it has an uncountable equilateral set.
\end{abstract}

\section{INTRODUCTION}

In this note we deal with the possible behaviour of the norm on uncountable sets of vectors in nonseparable Banach spaces. Our examples and results refer to Banach spaces of the form $C(K)$ that is spaces of real-valued continuous functions on a compact Hausdorff space $K$ with the supremum norm. However, our example is a novelty also in the entire class of Banach spaces. Recall that for $r \in \mathbb{R}$ a set $A$ of a Banach space $X$ is $r$-separated if $\left\|v_{1}-v_{2}\right\|>r$ for distinct $v_{1}, v_{2} \in A$ and it is $r$-equilateral if $\left\|v_{1}-v_{2}\right\|=r$ for all distinct $v_{1}, v_{2} \in A$. A set is called equilateral if it is $r$-equilateral for some $r \in \mathbb{R}$.

A natural question considered in the literature is whether given a Banach space of big dimension there is a big equilateral set or there is a big 1-separated or $(1+\varepsilon)$ separated set in the unit sphere of the Banach space. The classical Riesz lemma gives the optimal answer for $(1-\varepsilon)$-separation in the finite dimensional case. In the infinite dimensional separable case Kottman showed in [15] the existence of an infinite 1-separated set and Elton with Odell improved it for $(1+\varepsilon)$-separation for some $\varepsilon>0$. However there are infinite dimensional Banach spaces without infinite equilateral sets as first shown by Terenzi ([24], see also [9]).

1991 Mathematics Subject Classification. 46B25, 46B26, 03E35, 03E50, 54D30, 54F15.

The research was supported by grant PVE Ciência sem Fronteiras - CNPq, process number 406239/2013-4 . 
Already Elton and Odell noted that $c_{0}(\Gamma)$ for uncountable $\Gamma$ has no uncountable $(1+\varepsilon)$-separated sets for some $\varepsilon$. It is clear however that it has an uncountable equilateral set. Our main result is to give the first example of a nonseparable Banach space with no uncountable equilateral set. This example is not absolute in the sense that the construction requires an additional set-theoretic assumption consistent with the usual axioms ZFC. The example is of the form $C(K)$, and we are able to show that in this class of Banach spaces an additional set-theoretic assumption is necessary. This undecidability is compatible with many results of this type which keep appearing in recent years in all areas of classical mathematics from operator algebras through homology theory to the elementary properties of the Lebesgue measure and integral, where the continuum hypothesis provides pathological examples while a certain hierarchy of axioms which starts with Martin's axiom with the negation of the continuum hypothesis provide nice structural theory based on canonical examples, selection principles, dichotomies etc.

Let us discuss now the content of the paper in more details. In Section 2 we define (Definition 2.1) a class of compact nonmetrizable spaces called connectedly split intervals which are obtained by splitting points of a subset $\left\{r_{\xi}: \xi<\kappa\right\}$ of the interval $[0,1]$. These are connected generalizations of the classical split interval (e.g., 8 and for totally disconnected similar generalizations see also [14 and 4]). We analyze their relatively simple structure by embedding them in the products of intervals. They depend of the choice of the splitting functions $f_{\xi}:[0,1] \backslash\left\{r_{\xi}\right\} \rightarrow[-1,1]$ which are continuous in their domains but normally do not extend to a continuous function on $[0,1]$ and which determine the way the point $r_{\xi}$ is split in the final space. For our examples the prototypical splitting function is $\sin \left(\frac{1}{r-r_{\xi}}\right)$. Section 2 contains also simple properties of such spaces (Proposition 2.3) and in particular a lemma (Lemma 2.4) about simple representation of elements of a norm dense subset of $C(K)$ for $K$ in our class. It is remarkable that all continuous functions can be approximated by very simplified combinations of functions generating the Banach algebra $C(K)$.

In Section 3, in Definition 3.1 we introduce anti-Ramsey sequences $\left(f_{\xi}\right)_{\xi<\omega_{1}}$ of splitting functions. Similar constructions were used in our papers [14] and [4] where we constructed Banach spaces without uncountable semibiorthogonal sequences or without specific uncountable biorthogonal sequences. However, for simplicity the present construction contains simple uncountable biorthogonal systems. This with Corollary 1 of $[18$ shows that our space $C(K)$ has an equivalent renorming in which it has an uncountable equilateral system. This also shows that the presence of such systems does not imply the existence of uncountable equilateral sets. We do not know if stronger consistent versions could be obtained where no renorming has uncountable equilateral set.

Using the representation lemma (2.4) we prove one of our main results (Theorem 3.3) that if $\left(f_{\xi}\right)_{\xi<\omega_{1}}$ is anti-Ramsey then the obtained $C(K)$ has no uncountable equilateral set. It follows from the work of Mercourakis and Vassiliadis in [19] that such spaces cannot have in their unit sphere an uncountable $(1+\varepsilon)$-separated set for any $\varepsilon>0$. On the other hand Kania and Kochanek proved in [10] that every nonseparable $C(K)$ space has an uncountable 1-separated set. Roughly speaking in our $C(K)$ spaces (like in the spaces of [14, 4]) among any uncountable set of vectors there are two vectors which are related as we wish, of course up to some limitations which are built in the definition of a connectedly split interval. This 
type of structures was first systematically considered probably in 22 by S. Shelah. Since then a remarkable progress was made, one of the biggest steps of which were achieved by removing special set-theoretic assumptions from some of such constructions using the anti-Ramsey coloring due to Todorcevic of [25]. It was applied in the context of Banach spaces first in the work 23. of Shelah and Steprans and later [28] of Wark (further advanced in [1] or in [17]). We believe that it is a natural tool (see also 27]) which one can try to use to construct an absolute example of a nonseparable Banach space without an uncountable equilateral set. However, a result of Todorcevic from [26 says that it is consistent that every nonseparable Banach space contains an uncountable biorthogonal system which gives by Corollary 1 of 18 a renorming in which it has an uncountable equilateral set. One could also mention here related results of [3] concerning general vector spaces.

It is immediate that if $K$ is totally disconnected, then $C(K)$ has a 2-equilateral set of the maximal possible cardinality i.e., of the density of $C(K)$. For this one just takes $\chi_{A}-\chi_{K \backslash A}$ when $A$ runs through all clopen subsets of the compact $K$. This shows that our $K$ has no nonmetrizable totally disconnected subspace. Such spaces were known to exist under special set-theoretic assumptions. It remains open if such spaces can be constructed without any extra assumption or it is consistent that every compact nonmetrizable $K$ has a nonmetrizable totally disconnected subspace (see [13]). It is in the proof of Proposition 2.3 that we use the Darboux property of continuous functions on connected spaces based on which we can prove later that our Banach space does not contain an uncountable equilateral set. This argument would not go through for our totally disconnected versions of the split interval considered in the papers [4, 14.

We also do not know if one can consistently construct a nonseparable space $C(K)$ where all renormings do not have uncountable equilateral sets. Such examples would be new examples of spaces nonisomorphic to $C(L)$ s for totally disconnected $L$. The first such examples were obtained only recently in [12] with $K$ very big (e.g. strongly rigid, see [20) and later with $K$ sequentially compact in [2, but the spaces like in this paper would lead to first countable examples. Recall that it is not even known if the ball in the nonseparable Hilbert space with the weak topology is such a compact space.

In Section 4 we show the consistency of the existence of anti-Ramsey sequence of splitting functions. This is done by a forcing argument which follows the lines of 14] and [4. This section can be skipped by the readers not familiar with forcing. The readers which are forcing curious but do not want to go through a systematic forcing course as in [16] may consult books like [5] or [29. Our forcing argument belongs to the simplest possible, it is a single forcing and we are interested in elementary properties of the generic object. This argument shows that our example is consistent both with the continuum hypothesis and its negation. It seem an interesting technical question if such an example can be obtained just from the continuum hypothesis, for example along the lines of [21].

Section 5 is devoted to proving that assuming Martin's axiom and the negation of the continuum hypothesis every nonseparable Banach space of the form $C(K)$ contains an uncountable equilateral set. This is based on the use of an uncountable intersecting family of closed pairs introduced in [19] which is equivalent by Theorem 1 of [19] to the existence of 2-equilateral uncountable set in the sphere of $C(K)$ and 
to the existence of a $(1+\varepsilon)$-separated set for some $\varepsilon>0$. It turns out that such an uncountable intersecting family is an antichain in a forcing notion used extensively e.g. in [11, so if it does not exist, some natural forcing notions are c.c.c. which allows us to use Martin's axiom. It should be added that it was known (see 26]) that Martin's axiom implies the existence of uncountable biorthogonal systems in nonseparable Banach spaces of the form $C(K)$ and hence an uncountable equilateral set in some renorming of the $C(K)$ by 18 .

The notation and terminology is relatively standard, concerning Banach spaces we follow the book [7] and concerning set theory [16]. The Banach spaces we consider are of the form $C(K)$ for $K$ compact, infinite and Hausdorff with the supremum norm \|\| . Sometimes we will also consider bounded discontinuous $g: K \rightarrow \mathbb{R}$, then we use the notation $\|g\|_{\infty}=\sup \{|g(x)|: x \in K\}$. When a metric $d$ is fixed on some set $M$, then $\operatorname{diam}(M)=\sup \{d(x, y): x \in M\}$. The author would like to thank Marek Cuth for careful reading of the preliminary version of this paper and spotting some inaccuracies.

\section{Connectedly Split intervals}

Given a cardinal $\kappa$ and functions $f_{\xi}:[0,1] \backslash\left\{r_{\xi}\right\} \rightarrow[-1,1]$ for some distinct $r_{\xi} \in[0,1]$ for $\xi<\kappa$ we may define a natural connected version of the split interval (see e.g. [8]) which can be naturally embedded in the product space $[0,1] \times[-1,1]^{\kappa}$. A classical prototype of such an $f_{\xi}$ is the Warsaw $\sin$ function i.e., $\sin \left(\frac{1}{r-r_{0}}\right)$ for some $r_{0} \in(0,1)$.

Definition 2.1. Let $\kappa \leq 2^{\omega}$ be a cardinal. Suppose that $\left\{r_{\xi}: \xi<\kappa\right\}$ consists of distinct elements of $[0,1]$ and $f_{\xi}:[0,1] \backslash\left\{r_{\xi}\right\} \rightarrow[-1,1]$ is a continuous function for every $\xi<\kappa$. A connectedly split interval induced by $\left(f_{\xi}\right)_{\xi<\kappa}$ is the subspace $K$ of $[0,1]^{\{*\}} \times[-1,1]^{\kappa}$ consisting of points of the form

$$
\left\{x_{\xi, t},: \xi<\kappa, t \in[-1,1]\right\} \cup\left\{x_{r}: r \in[0,1] \backslash\left\{r_{\xi}: \xi<\kappa\right\}\right\},
$$

where

(1) $x_{\xi, t}(*)=r_{\xi}, x_{\xi, t}(\xi)=t$ and $x_{\xi, t}(\eta)=f_{\eta}\left(r_{\xi}\right)$ if $\eta \in \kappa \backslash\{\xi\}$,

(2) $x_{r}(*)=r$ and $x_{r}(\xi)=f_{\xi}(r)$ for all $r \in[0,1] \backslash\left\{r_{\xi}: \xi<\kappa\right\}$ and $\xi<\kappa$.

Under these assumptions we will use the following notation and terminology:

(1) $\overline{f_{*}}: K \rightarrow[0,1]$ is given by $\overline{f_{*}}(x)=x(*)$ for any $x \in K$,

(2) $\overline{f_{\xi}}: K \rightarrow[0,1]$ is given by $\overline{f_{\xi}}(x)=x(\xi)$ for all $\xi<\kappa$ and $x \in K$.

(3) $(a, b)_{K}=\{x \in K: a<x(*)<b\}$, and similar meaning for $(a, b]_{K},[a, b)_{K}$, $[a, b]_{K}$ for all $0 \leq a<b \leq 1$.

(4) $(a, b)_{K, \xi}=\{x \in K: a<x(\xi)<b\}$, and similar meaning for $(a, b]_{K, \xi}$, $[a, b)_{K, \xi},[a, b]_{K, \xi}$ for all $0 \leq a<b \leq 1$ and every $\xi<\kappa$.

(5) $R_{\xi}=\left\{\left[x_{\xi, t}\right]: t \in[-1,1]\right\}$ for all $\xi<\kappa$,

(6) $f_{\xi}$ s will be called splitting functions.

The coordinate in the product $[0,1]^{\{*\} \cup \kappa}$ corresponding to $*$ will be called the *-coordinate.

Thus, the classical split interval $S$ is obtained by choosing $(0,1)=\left\{r_{\xi}: \xi<2^{\omega}\right\}$, $f_{\xi}:[0,1] \backslash\left\{r_{\xi}\right\}$ defined by $f_{\xi}(r)=0$ if $r<r_{\xi}$ and $f_{\xi}(r)=1$ if $r>r_{\xi}$ and considering the subspace $S=K \cap\left([0,1]^{\{*\}} \times\{0,1\}^{2^{\omega}}\right)$ of the connectedly split interval $K$ induced by $\left(f_{\xi}\right)_{\xi<2^{\omega}}$. Note that it follows from the definition of the connectedly split interval $K$ that the only point $x$ of $K$ such that $x(*)=r$ is $x_{r}$ 
if $r \in[0,1] \backslash\left\{r_{\xi}: \xi<\kappa\right\}$ and that the only points $x$ of $K$ such that $x(*)=r_{\xi}$ for $\xi<\kappa$ are the points of $R_{\xi}$ that is $x_{\xi, t}$ for $t \in[-1,1]$ and these points differ just at the $\xi$-th coordinate and are equal on all other coordinates of the product.

Lemma 2.2. Suppose that $K$ is Hausdorff compact and connected, $x_{0} \in K$ and $f: K \backslash\left\{x_{0}\right\} \rightarrow[-1,1]$ is continuous. Then

$$
L=\left\{(x, t) \in K \times[-1,1]: x \neq x_{0} \Rightarrow t=f(x)\right\}
$$

is compact and connected.

Proof. If $(x, t) \in(K \times[-1,1]) \backslash L$, then $x \neq x_{0}$ and there is $\varepsilon>0$ such that $|f(x)-t|>\varepsilon$. By the continuity of $f$ at $x$ there is a neighbourhood $U$ of $x$ such that $x_{0} \notin U$ and $\left|f\left(x_{1}\right)-f\left(x_{0}\right)\right|<\varepsilon / 2$ for all $x_{1} \in U$. It follows that $U \times(t-\varepsilon / 2, t+\varepsilon / 2)$ is disjoint from $L$ and contains $(x, t)$, hence the complement of $L$ is open and so $L$ is compact.

For connectedness suppose that $L \subseteq A \cup B$ where $A, B \subseteq K \times[-1,1]$ are open with disjoint closures and $L \cap A \neq \emptyset \neq L \cap B$. Define $A^{\prime} \subseteq K$ to be the set of $x \in$ $K \backslash\left\{x_{0}\right\}$ such that $(x, f(x)) \in A$ and $B^{\prime} \subseteq K$ to be the set of $x \in K \backslash\left\{x_{0}\right\}$ such that $(x, f(x)) \in B$. By the continuity of $f$ the sets $B^{\prime}$ and $A^{\prime}$ are disjoint open subsets of $K$ such that $A^{\prime} \cup B^{\prime}=K \backslash\left\{x_{0}\right\}$. We may assume that $\left(\left\{x_{0}\right\} \times[-1,1]\right) \cap A \neq \emptyset$, and so by the connectedness of $[-1,1]$ we have $\left\{x_{0}\right\} \times[-1,1] \subseteq A$ and by the compactness of $[-1,1]$ we have a neighbourhood $U$ of $x_{0}$ such that $U \times[-1,1] \subseteq A$. Thus $A^{\prime} \cup\left\{x_{0}\right\}$ contains $U$ and so is open and hence the partition of $K$ into $A^{\prime} \cup\left\{x_{0}\right\}$ and $B^{\prime}$ contradicts the connectedness of $K$.

Proposition 2.3. Suppose that $\left\{r_{\xi}: \xi<\kappa\right\} \subseteq[0,1]$ where $\kappa$ is an uncountable cardinal. Suppose that $K \subseteq[0,1]^{\{*\}} \times[-1,1]^{\kappa}$ is a generalized split interval induced by $\left(f_{\xi}\right)_{\xi<\kappa}$ where $f_{\xi}:[0,1] \backslash\left\{r_{\xi}\right\} \rightarrow[-1,1]$. Then

(1) $K$ is a compact Hausdorff space of weight $\kappa$,

(2) $K$ is connected,

(3) $K$ is first countable,

$$
\left\{(r-1 / n: r+1 / n)_{K}: n \in \mathbb{N}\right\}
$$

forms a basis at $x_{r}$ for each $r \in[0,1] \backslash\left\{r_{\xi}: \xi<\kappa\right\}$, and

$$
\left\{\left(r_{\xi}-1 / n: r_{\xi}+1 / n\right)_{K} \cap(t-1 / n, t+1 / n)_{K, \xi}: n \in \mathbb{N}\right\}
$$

forms a basis at $x_{\xi, t}$ for each $t \in[-1,1]$ and each $\xi<\kappa$,

(4) $K^{2}$ has a discrete set of cardinality $\kappa$,

(5) $C(K)$ has a biorthogonal system of cardinality $\kappa$.

(6) $\overline{f_{\xi}} \uparrow\left(K \backslash R_{\xi}\right)=f_{\xi} \circ \overline{f_{*}} \uparrow\left(K \backslash R_{\xi}\right)$

Proof. (1) If $x \in[0,1] \times[-1,1]^{\kappa}$ is not in $K$ it is because either $x(*)=r_{\xi}$ for some $\xi<\kappa$ but $f_{\eta}\left(r_{\xi}\right) \neq x(\eta)$ for some $\xi \neq \eta<\kappa$ or $x(*) \notin\left\{r_{\xi}: \xi<\kappa\right\}$ and $f_{\eta}(x(*)) \neq x(\eta)$ for some $\eta<\kappa$. In both cases one sees that these conditions define open sets in $[0,1]^{\{*\}} \times[-1,1]^{\kappa}$.

(2) We can see $K$ as the inverse limit of $K_{\eta}$ for $\eta<\kappa$ where $K_{\eta} \subseteq[0,1]^{\{*\}} \times$ $[-1,1]^{\eta}$ is a connectedly split interval induced by $\left(f_{\xi}\right)_{\xi<\eta}$. As inverse limits of compact connected spaces are compact connected, it is enough to prove that $K_{\eta+1}$ is connected provided $K_{\eta}$ is. This follows from Lemma 2.2 since by the definition of the connectedly split interval $K_{\eta}$ the point $x_{\eta, t} \uparrow \eta=\widetilde{r_{\eta}} f_{\xi}\left(r_{\eta}\right)_{\xi<\eta}$ (for any $t \in[-1,1])$ is the unique point of $K_{\eta}$ such that its projection on the $*$-coordinate 
is $r_{\eta}$, so $K_{\eta+1}$ is obtained from $K_{\eta}$ as in Lemma 2.2 for $f: K_{\eta} \backslash x_{\eta, 0}\lceil\eta \rightarrow[-1,1]$ defined by $f(x)=f_{\eta}(x(*))$ for any $x$ in the domain of $f$.

(3) The mentioned collections of neighbourhoods define decreasing pseudobases at indicated points, but such pseudobases are bases in compact spaces.

(4) Consider $\left(x_{\xi,-1}, x_{\xi, 1}\right) \in K \times K$ and open $U_{\xi} \times V_{\xi} \subseteq K \times K$ given by $U_{\xi}=\{x \in$ $K: x(\xi)<0\}$ and $V_{\xi}=\{x \in K: x(\xi)>0\}$. Note that $x_{\xi, 1}(\eta)=x_{\xi,-1}(\eta)=f_{\eta}\left(r_{\xi}\right)$ for any $\xi \neq \eta<\kappa$, and so $\left(x_{\xi,-1}, x_{\xi, 1}\right) \in U_{\eta} \times V_{\eta}$ if and only if $\xi=\eta$ meaning that $\left(x_{\xi,-1}, x_{\xi, 1}\right)_{\xi<\kappa}$ is discrete.

(5) Define $g_{\xi}=\overline{f_{\xi}} / 2$ and $\mu_{\xi}=\delta_{x_{\xi, 1}}-\delta_{x_{\xi,-1}}$. We have

$$
\int g_{\xi} d \mu_{\xi}=\frac{1}{2}\left(\overline{f_{\xi}}\left(x_{\xi, 1}\right)-\overline{f_{\xi}}\left(x_{\xi,-1}\right)\right)=\frac{1}{2}(1-(-1))=1
$$

and

$$
\int g_{\xi} d \mu_{\eta}=\frac{1}{2}\left(\overline{f_{\xi}}\left(x_{\eta, 1}\right)-\overline{f_{\xi}}\left(x_{\eta,-1}\right)\right)=\frac{1}{2}\left(f_{\xi}\left(r_{\eta}\right)-f_{\xi}\left(r_{\eta}\right)\right)=0
$$

if $\xi \neq \eta<\kappa$.

Connectedly split intervals may or may not be hereditarily separable or hereditarily Lindelöf. This depends on the sequence of $f_{\xi}$ s. The split intervals of Section 3 will be hereditarily Lindelöf and hereditarily separable.

By a piecewise polynomial with rational coefficients we will mean a function (not necessarily continuous) whose domain $[0,1]$ can be divided into finitely many intervals on which the function is equal to a polynomial with rational coefficients. By a rational polynomial we mean a polynomial with rational coefficients which assumes all its local minima and maxima in rational points. It is clear that rational polynomials on a bounded interval form a dense set of continuous functions of this interval, this could be observed by writing the derivative of an arbitrary polynomial as the product of linear and indecomposable quadratic factors and adjusting slightly the roots of the linear factors so that the integral of the derivative is not affected much.

Lemma 2.4. Suppose that $\left\{r_{\xi}: \xi<\kappa\right\} \subseteq[0,1]$ where $\kappa$ is an uncountable cardinal. Suppose that $K \subseteq[0,1]^{\{*\}} \times[-1,1]^{\kappa}$ is a connectedly split interval induced by $\left(f_{\xi}\right)_{\xi<\kappa}$ where $f_{\xi}:[0,1] \backslash\left\{r_{\xi}\right\} \rightarrow[-1,1]$. Let $\varepsilon>0$ and $f \in C(K)$, then there is a (possibly discontinuous) function $g: K \rightarrow \mathbb{R}$ of the form

$$
g=P_{-1} \circ \overline{f_{*}}+\Sigma_{i \leq k} P_{i}\left(\overline{f_{\xi_{i}}}\right) \chi_{\left(s_{i}, s_{i}^{\prime}\right)_{K}}
$$

where $k \in \mathbb{N}, \xi_{1}, \ldots, \xi_{k}<\kappa, P_{-1}$ is a piecewise polynomial in one variable with rational coefficients, $P_{i}$ for $1 \leq i \leq k$ are rational polynomials in one variable, $s_{i}, s_{i}^{\prime}$ are rationals such that $r_{\xi_{i}} \in\left(s_{i}, s_{i}^{\prime}\right)$ and the intervals $\left(s_{i}, s_{i}^{\prime}\right) s$ for $1 \leq i \leq k$ are pairwise disjoint, and

$$
\|f-g\|_{\infty}<\varepsilon .
$$

Proof. Recall from Definition 2.1 that $\overline{f_{\xi}}(x)=x(\xi)$ for $\xi<\kappa$ and $\overline{f_{*}}(x)=x(*)$ for all $x \in K$. The coordinates of $K \subseteq[0,1]^{\{*\}} \times[-1,1]^{\kappa}$ separate the points of $K$, so by the Stone-Weierstrass theorem every function in $C(K)$ can be approximated by a function from the algebra generated by the constant functions and $\overline{f_{\xi}}$ s for $\xi<\kappa$ and by $\overline{f_{*}}$. In this algebra the functions of the form

$$
Q\left(\overline{f_{*}}, \overline{f_{\xi_{1}}}, \ldots, \overline{f_{\xi_{k}}}\right)
$$


are dense where $Q$ is a polynomial in $(k+1)$-variables with rational coefficients and $\xi_{1}<\ldots<\xi_{n}$ for some $k \in \mathbb{N}$. So we may assume that $f=Q\left(\overline{f_{*}}, \overline{f_{\xi_{1}}}, \ldots, \overline{f_{\xi_{k}}}\right)$ for one of such polynomials.

Note that for $\xi<\kappa$ we have $\overline{f_{\xi}}(x)=f_{\xi}(x(*))=f_{\xi}\left(\overline{f_{*}}(x)\right)$ for $x \in K$ outside of the intervals $(a, b)_{K}$ for $0 \leq a<r_{\xi}<b \leq 1$ and that $f_{\xi}:[0,1] \backslash\left\{r_{\xi}\right\} \rightarrow[-1,1]$ is continuous outside $(a, b)$ for such $a, b$. It follows that around $r_{\xi_{i}}$ there are intervals $\left(s_{i}, s_{i}^{\prime}\right) \ni r_{\xi_{i}}$ with rational endpoints where the oscillations of $f_{\xi_{j}}$ for $j \neq i$ and $1 \leq j \leq k$ is arbitrarily small. So for each $1 \leq i \leq k$ find such an interval $\left(s_{i}, s_{i}^{\prime}\right) \ni r_{\xi_{i}}$ that

$$
\left|Q\left(\bar{f}_{*}, \bar{f}_{\xi_{1}}, \ldots, \bar{f}_{x_{\xi_{i}}}, \ldots, \bar{f}_{\xi_{k}}\right)(x)-Q\left(q_{i, *}, q_{i, 1}, \ldots, \bar{f}_{x_{\xi_{i}}}, \ldots, q_{i, k}\right)(x)\right|<\varepsilon
$$

for some rationals $\left\{q_{i, *}, q_{i, j}:-1 \leq j \leq k, j \neq i\right\}$ and all $x \in\left(s_{i}, s_{i}^{\prime}\right)_{K}$. Let $P_{i}(v)=Q\left(q_{i, *}, q_{i, 1}, \ldots, v, \ldots, q_{i, n}\right)$ be the corresponding polynomial in one variable $v$, that is for all $x \in\left(s_{i}, s_{i}^{\prime}\right)_{K}$ we have

$$
\left|f(x)-P_{i}\left(\bar{f}_{x_{\xi_{i}}}\right)(x)\right|<\varepsilon .
$$

We may assume it is a rational polynomial by the density of such polynomials. Outside of the intervals $\left(s_{i}, s_{i}^{\prime}\right)_{K}$ the function $f=Q$ depends only on the $*$-coordinate and so it can be approximated by a polynomial in the $*$-coordinate with rational coefficients. Joining together these polynomials with the zero function on the intervals $\left(s_{i}, s_{i}^{\prime}\right)$ for $1 \leq i \leq k$ we obtain a piecewise polynomial $P_{-1}$ in one variable with rational coefficients such that

$$
\left|f(p)-P_{-1} \circ \overline{f_{*}}(p)\right|<\varepsilon
$$

for all $p \in K \backslash \bigcup_{1 \leq i \leq n}\left(s_{i}, s_{i}^{\prime}\right)_{K}$. This completes the proof.

\section{An ANTi-RAmSEy SEQUENCE OF SPLitting FUnCtions}

Definition 3.1. Suppose that $\left\{r_{\xi}: \xi<\kappa\right\} \subseteq[0,1]$ where $\kappa$ is an uncountable cardinal. Suppose that $f_{\xi}:[0,1] \backslash\left\{r_{\xi}\right\} \rightarrow[0,1]$ are continuous in their domains. We say that the sequence $\left(f_{\xi}\right)_{\xi<\kappa}$ is anti-Ramsey if given:

(a) $m \in \mathbb{N}$,

(b) pairwise disjoint nonempty open intervals $I_{1}, \ldots, I_{m}$ of $[0,1]$ with rational endpoints,

(c) any uncountable sequence $\left(F_{\alpha}\right)_{\alpha<\omega_{1}}$ where $F_{\alpha}=\left\{\xi_{1}^{\alpha}, \ldots, \xi_{m}^{\alpha}\right\}$ are pairwise disjoint finite subsets of $\kappa$ such that $r_{\xi_{i}^{\alpha}} \in I_{i}$ for every $1 \leq i \leq m$ and every $\alpha<\omega_{1}$,

(d) any two $m$-tuples $\left\{q_{1}, \ldots, q_{m}\right\}$ and $\left\{q_{1}^{\prime}, \ldots, q_{m}^{\prime}\right\}$ of rationals from $[-1,1]$

there are $\alpha<\beta<\omega_{1}$ and open intervals $\left(J_{i}^{\alpha}\right)_{1 \leq i \leq m}$, and $\left(J_{i}^{\beta}\right)_{1 \leq i \leq m}$ such that for each $1 \leq i \leq m$ we have:

(1) $J_{i}^{\alpha}, J_{i}^{\beta} \subseteq I_{i} ; J_{i}^{\alpha} \cap J_{i}^{\beta}=\emptyset$,

(2) $r_{\xi_{i}^{\alpha}} \in J_{i}^{\alpha}$ and $r_{\xi_{i}^{\beta}} \in J_{i}^{\beta}$,

(3) $f_{\xi_{i}^{\alpha}} \uparrow\left([0,1] \backslash\left(J_{i}^{\alpha} \cup J_{i}^{\beta}\right)\right)=f_{\xi_{i}^{\beta}} \uparrow\left([0,1] \backslash\left(J_{i}^{\alpha} \cup J_{i}^{\beta}\right)\right)$,

(4) $f_{\xi_{i}^{\alpha}} \uparrow J_{i}^{\beta}=q_{i}$,

(5) $f_{\xi_{i}^{\beta}} \uparrow J_{i}^{\alpha}=q_{i}^{\prime}$.

Proposition 3.2. Suppose that $\left\{r_{\xi}: \xi<\kappa\right\} \subseteq[0,1]$ where $\kappa$ is an uncountable cardinal. Suppose that $K \subseteq[0,1]^{\{*\}} \times[-1,1]^{\kappa}$ is connectedly split interval induced 
by an anti-Ramsey sequence of functions $\left(f_{\xi}\right)_{\xi<\kappa}$ where $f_{\xi}:[0,1] \backslash\left\{r_{\xi}\right\} \rightarrow[0,1]$. Suppose that $\left(g_{\alpha}\right)_{\alpha<\omega_{1}}$ is is a sequence of functions in $C(K)$ of the form

$$
g_{\alpha}=\Sigma_{i \leq m} P_{i}\left(\overline{f_{\xi_{i}^{\alpha}}}\right) \chi_{\left(s_{i}, s_{i}^{\prime}\right)_{K}}
$$

where

- $F_{\alpha}=\left\{\xi_{1}^{\alpha}, \ldots, \xi_{m}^{\alpha}\right\}$ are pairwise disjoint finite subsets of $\omega_{1}$ for $\alpha<\omega_{1}$,

- $P_{i} s$ are rational polynomials and $P_{i}[[-1,1]]=\left[a_{i}, b_{i}\right]$ for some $a_{i}, b_{i} \in \mathbb{R}$

- $\max \left\{\left|b_{i}-a_{i}\right|: 1 \leq i \leq m\right\}=\delta>0$,

- $s_{i}, s_{i}^{\prime}$ are rationals such that $r_{\xi_{i}^{\alpha}} \in\left(s_{i}, s_{i}^{\prime}\right)$ and $\left(s_{i}, s_{i}^{\prime}\right) s$ for $1 \leq i \leq m$ are pairwise disjoint

Then there are $\alpha<\beta<\omega_{1}$ such that $\left\|g_{\alpha}-g_{\beta}\right\|_{\infty}=\delta / 2$ and there are $\alpha^{\prime}<\beta^{\prime}<\omega_{1}$ such that $\left\|g_{\alpha^{\prime}}-g_{\beta^{\prime}}\right\|_{\infty}=\delta$.

Proof. To find $\alpha<\beta<\omega_{1}$ such that $\left\|g_{\alpha}-g_{\beta}\right\|=\delta / 2$ for every $1 \leq i \leq m$ find $q_{i}=q_{i}^{\prime} \in[-1,1]$ such that $P_{i}\left(q_{i}\right)$ is the middle point of $\left[a_{i}, b_{i}\right]$ and apply Definition 3.1 for $I_{i}=\left(s_{i}, s_{i}^{\prime}\right)$. As $P_{i}$ s are rational polynomials, i.e., with rational coefficients and with local maxima and minima in rational points, $q_{i}$ s are rational as required in 3.1. Here we also use the connectedness of $[-1,1]$, that is, this point would not go through if we had the Cantor set as in the papers [14, 4] instead of $[-1,1]$. By (3) of Definition 3.1 the functions $g_{\alpha}$ and $g_{\beta}$ may only differ on $J_{i}^{\alpha}$ and $J_{i}^{\beta}$ for $1 \leq i \leq m$, actually

$\left.\left.\left\|g_{\alpha}-g_{\beta}\right\|_{\infty}=\max _{1 \leq i \leq m}\left(\max _{x \in\left(J_{i}^{\alpha}\right)_{K}} \mid P_{i}\left(\overline{f_{\xi_{i}^{\alpha}}}\right)(x)\right)-P_{i}\left(q_{i}\right)\left|, \max _{x \in\left(J_{i}^{\beta}\right)_{K}}\right| P_{i}\left(\overline{f_{\xi_{i}^{\beta}}}(x)\right)-P\left(q_{i}^{\prime}\right)\right) \mid\right)$

As $\overline{f_{\xi_{i}^{\alpha}}}$ and $\overline{f_{\xi_{i}^{\beta}}}$ have the range $[-1,1]$ and assume all these values in $R_{\xi_{i}^{\alpha}} \subseteq\left(J_{i}^{\alpha}\right)_{K}$ and $R_{\xi_{i}^{\beta}} \subseteq\left(J_{i}^{\delta}\right)_{K}$ respectively we conclude that $\left\|g_{\alpha}-g_{\beta}\right\|=\delta / 2$ by the choice of $q_{i}=q_{i}^{\prime}$ for all $1 \leq i \leq k$.

To find $\alpha^{\prime}<\beta^{\prime}<\omega_{1}$ such that $\left\|g_{\alpha^{\prime}}-g_{\beta^{\prime}}\right\|=\delta$ for every $1 \leq i \leq k$ find $q_{i}=q_{i}^{\prime} \in[-1,1]$ such that $P_{i}\left(q_{i}\right)$ is the minimum point of $\left[a_{i}, b_{i}\right]$ that is $P_{i}\left(q_{i}\right)=a_{i}$ and apply Definition 3.1 for $I_{i}=\left(s_{i}, s_{i}^{\prime}\right)$. By (3) of Definition $3.1 g_{\alpha}$ and $g_{\beta}$ may only differ on $J_{i}^{\alpha}$ and $J_{i}^{\beta}$ for $1 \leq i \leq m$, actually

$\left.\left.\left\|g_{\alpha}-g_{\beta}\right\|_{\infty}=\max _{1 \leq i \leq m}\left(\max _{x \in\left(J_{i}^{\alpha}\right)_{K}} \mid P_{i}\left(f_{\xi_{i}^{\alpha}}\right)(x)\right)-P_{i}\left(q_{i}\right)\left|, \max _{x \in\left(J_{i}^{\beta}\right)_{K}}\right| P_{i}\left(f_{\xi_{i}^{\beta}}(x)\right)-P_{i}\left(q_{i}^{\prime}\right)\right) \mid\right)$

As $f_{\xi_{i}^{\alpha}}$ and $f_{\xi_{i}^{\beta}}$ have the range $[-1,1]$ and assume all these values in $R_{\xi_{i}^{\alpha}} \subseteq\left(J_{i}^{\alpha}\right)_{K}$ and $R_{\xi_{i}^{\beta}} \subseteq\left(J_{i}^{\delta}\right)_{K}$ respectively we conclude that $\left\|g_{\alpha}-g_{\beta}\right\|=\max _{1 \leq i \leq k}\left|b_{i}-a_{i}\right|=\delta$ by considering $x \in R_{\xi_{i}^{\alpha}}$ where $\left.P_{i}\left(f_{\xi_{i}^{\alpha}}\right)(x)\right)=b_{i}$ by the choice of $q_{i}$ for all $1 \leq i \leq$ $k$.

Theorem 3.3. Suppose that $\left\{r_{\xi}: \xi<\omega_{1}\right\} \subseteq[0,1]$ Suppose that $K \subseteq[0,1]^{\{*\}} \times$ $[-1,1]^{\kappa}$ is a connectedly split interval induced by an anti-Ramsey sequence of functions $\left(f_{\xi}\right)_{\xi<\omega_{1}}$ where $f_{\xi}:[0,1] \backslash\left\{r_{\xi}\right\} \rightarrow[-1,1]$. Then $C(K)$ has no uncountable equilateral set nor an uncountable $(1+\varepsilon)$-separated set in the unit ball.

Proof. Let $\left\{e_{\alpha}: \alpha<\omega_{1}\right\}$ be a collection of distinct elements of the unit ball of $C(K)$. Let $\delta \in \mathbb{R}$ be the maximal real such that for every $\alpha<\omega_{1}$, for every $\varepsilon>0$ there are $\alpha<\beta, \xi<\omega_{1}$ such that $\operatorname{diam}\left(e_{\beta}\left[R_{\xi}\right]\right)>\delta-\varepsilon$. As the $e_{\alpha} \mathrm{s}$ are from the unit ball it is clear that $\delta \leq 2$.

Now let us note we may assume that $\delta>0$ : otherwise there is $\alpha<\omega_{1}$ such that for every $\alpha<\beta, \xi<\omega_{1} \operatorname{diam}\left(e_{\beta}\left[R_{\xi}\right]\right)=0$; but two points $x, y \in K$ of a connectedly 
split interval which first differ at a coordinate above $\alpha$ must both belong to some $R_{\xi}$ for $\xi>\alpha$. This means that for $\beta>\alpha$ the functions $e_{\beta}$ depend only on the countably many coordinates from $\{*\} \cup[0, \alpha]$, but $C\left([0,1] \times[-1,1]^{\alpha+1}\right)$ is a separable Banach space so among $e_{\alpha}$ s for $\alpha<\omega_{1}$ there are functions arbitrarily close to each other and so they cannot form an equilateral set. Thus we may assume that $\delta>0$ indeed.

By Lemma 2.4 we can approximate each $e_{\alpha}$ by a function $g_{\alpha}^{\prime}$ of the form specified in that lemma with $\left\|e_{\alpha}-g_{\alpha}^{\prime}\right\|_{\infty}<\frac{\delta}{20}$. Choosing $\varepsilon=\frac{\delta}{20}$ above we conclude that for arbitrarily high $\beta, \xi<\omega_{1}$ we have $\operatorname{diam}\left(g_{\beta}^{\prime}\left[R_{\xi}\right]\right) \in\left(\frac{19}{20} \delta, \frac{21}{20} \delta\right)$. Using the $\Delta$-system lemma and the fact that there are only countably many polynomials with rational coefficients and countably many intervals with rational endpoints and using the maximality of $\delta$ by going to a subsequence we may assume that for every $\alpha<\omega_{1}$

$$
g_{\alpha}^{\prime}=P_{-1} \circ \overline{f_{*}}+\Sigma_{1 \leq i \leq m} P_{i}\left(\overline{f_{\xi_{i}^{\alpha}}}\right) \chi_{\left(s_{i}, s_{i}^{\prime}\right)}+\Sigma_{m<i \leq k} P_{i}\left(\overline{f_{\xi_{i}}}\right) \chi_{\left(s_{i}, s_{i}^{\prime}\right)}
$$

where

- $F_{\alpha}=\left\{\xi_{1}^{\alpha}, \ldots, \xi_{m}^{\alpha}\right\}$ are pairwise disjoint finite subsets of $\omega_{1}$ for $\alpha<\omega_{1}$ all disjoint from $\left\{\xi_{m+1}, \ldots, \xi_{k}\right\}$,

- $P_{-1}$ is a piecewise polynomial with rational coefficients,

- $P_{i}$ s for $1 \leq i \leq k$ are rational polynomials,

- $s_{i}, s_{i}^{\prime}$ are rationals such that $r_{\xi_{i}^{\alpha}} \in\left(s_{i}, s_{i}^{\prime}\right)$ for $1 \leq i \leq m$ and $r_{\xi_{i}} \in\left(s_{i}, s_{i}^{\prime}\right)$ for $m<i \leq k$ and

- $\left(s_{i}, s_{i}^{\prime}\right) \mathrm{s}$ for $1 \leq i \leq k$ are pairwise disjoint,

- $P_{i}[[-1,1]]=\left[a_{i}, b_{i}\right]$ for some $a_{i}, b_{i} \in \mathbb{R}$ and all $1 \leq i \leq k$,

- $\max \left\{\left|b_{i}-a_{i}\right|: 1 \leq i \leq m\right\} \in\left(\frac{19}{20} \delta, \frac{21}{20} \delta\right)$.

The last item follows from the fact that $\operatorname{diam}\left(g_{\alpha}^{\prime}\left[R_{\xi}\right]\right) \in\left(\frac{19}{20} \delta, \frac{21}{20} \delta\right)$ for some $\xi<\omega_{1}$ and $\overline{f_{\xi}}$ separates points of $R_{\xi^{\prime}}$ if and only if $\xi=\xi^{\prime}$. For $\alpha<\omega_{1}$ put

$$
g_{\alpha}=\Sigma_{1 \leq i \leq m} P_{i}\left(\overline{f_{\xi_{i}^{\alpha}}}\right) \chi_{\left(s_{i}, s_{i}^{\prime}\right)}
$$

and note that $g_{\alpha}-g_{\beta}=g_{\alpha}^{\prime}-g_{\beta}^{\prime}$ for every $\alpha, \beta<\omega_{1}$. Using Proposition 3.2 we find $\alpha<\beta<\omega_{1}$ such that $\left\|g_{\alpha}-g_{\beta}\right\| \leq \frac{11}{20} \delta$ and $\alpha^{\prime}<\beta^{\prime}<\omega_{1}$ such that $\left\|g_{\alpha^{\prime}}-g_{\beta^{\prime}}\right\| \geq \frac{18}{20} \delta$. So $\left\|e_{\alpha}-e_{\beta}\right\| \leq \frac{13}{20} \delta$ and $\left\|e_{\alpha}-e_{\beta^{\prime}}\right\| \geq \frac{16}{20} \delta$ which shows that the functions $e_{\alpha}$ s do not form an equilateral set. The last part of the statement of the theorem follows from Theorem 1 of [19.

\section{ForCinG}

In this section we use the method of forcing to show the consistency of the existence of an anti-Ramsey sequence of functions $\left(f_{\xi}\right)_{\xi<\omega_{1}}$.

Definition 4.1. Let $0<a<b<1$. We say that $f:[0, a] \cup[b, 1] \rightarrow[-1,1]$ is rationally piecewise linear if it is continuous on $[0, a] \cup[b, 1]$ and there are pairwise disjoint intervals $\left(I_{i}\right)_{i \leq k}$ with rational endpoints for $1 \leq i \leq k \in \mathbb{N}$ such that $[0, a] \cup[b, 1]=\bigcup_{1 \leq i \leq k} I_{i}$ and $f(r)=a_{i} r+b_{i}$ for all $r \in I_{i}$ and some rationals $a_{i}, b_{i}$ and each $1 \leq i \leq \bar{k}$.

Rationally piecewise linear functions defined on sets $[0, r-\varepsilon] \cup[r+\varepsilon, 1]$ for some $\varepsilon>0$ and $r \in(0,1)$ will serve for us as approximations for splitting functions $f:[0,1] \backslash\{r\} \rightarrow[-1,1]$. We construct a sequence of splitting functions in the generic extension of any set-theoretic universe obtained with the following forcing notion: 
Definition 4.2. Fix an uncountable set $\left\{r_{\xi}: \xi<\omega_{1}\right\}$ of irrationals. $\mathbb{P}$ is a forcing notion consisting of triples $\left(n_{p}, F_{p}, \mathcal{F}_{p}\right)$ such that:

(1) $n_{p} \in \mathbb{N}$,

(2) $F_{p} \subseteq \omega_{1}$ is a finite set, such that there is an injective function sending $F_{p} \ni \xi \rightarrow k_{\xi} \in\left\{k \in \mathbb{N}: 0 \leq k<2^{n_{p}}\right\}$ such that $r_{\xi} \in\left(\frac{k_{\xi}}{2^{n_{p}}}, \frac{k_{\xi}+1}{2^{n_{p}}}\right)$,

(3) $\mathcal{F}_{p}=\left\{f_{p}^{\xi}: \xi \in F_{p}\right\}$,

(4) $f_{p}^{\xi}:\left[0, \frac{k_{\xi}}{2^{n_{p}}}\right] \cup\left[\frac{k_{\xi}+1}{2^{n_{p}}}, 1\right] \rightarrow[-1,1]$ is a rationally piecewise linear function for each $0 \leq k<2^{n_{p}}$.

We say that $p \leq q$ if and only if

(a) $n_{p} \geq n_{q}$,

(b) $F_{p} \supseteq F_{q}$,

(c) $f_{p}^{\xi} \supseteq f_{q}^{\xi}$ for every $\xi \in F_{q}$.

The motivation of the above definition should be clear. It is the partial order of approximations to the anti-Ramsey sequence of functions as in Definition 3.1 a given condition $p \in \mathbb{P}$ provides approximations to finitely many $f_{\xi} \mathrm{s}$ for $\xi \in F_{p}$ in the form of a rationally piecewise linear function $f_{p}^{\xi}:\left[0, \frac{k_{\xi}}{2^{n_{p}}}\right] \cup\left[\frac{k_{\xi}+1}{2^{n_{p}}}, 1\right] \rightarrow[-1,1]$. The density argument in Proposition 4.4 will allow us to prove that the approximated sequence of splitting functions is anti-Ramsey. First we need to take care of the preservation of the cardinals.

Lemma 4.3. $\mathbb{P}$ satisfies the c.c.c.

Proof. Suppose that $\left(p_{\alpha}: \alpha<\omega_{1}\right)$ consists of distinct elements of $\mathbb{P}$. We will find $\alpha<\beta<\omega_{1}$ and $o \in \mathbb{P}$ such that $o \leq p_{\alpha}, p_{\beta}$. Using the fact that there are countably many finite systems of rationals and countably many rationally piecewise linear functions by going to an uncountble subsequence we may assume that the following holds for all $\alpha<\omega_{1}$ :

- $n_{p_{\alpha}}=n$ for some $n \in \mathbb{N}$,

- $\left(F_{p_{\alpha}}\right)_{\alpha<\omega_{1}}$ forms a $\Delta$-system $F_{p_{\alpha}}=\Delta \cup\left\{\xi_{1}^{\alpha}, \ldots, \xi_{m}^{\alpha}\right\}$ with root $\Delta=\left\{\xi_{1}, \ldots, \xi_{l}\right\}$,

- there are distinct $k_{1}^{\prime}, \ldots, k_{l}^{\prime}, k_{1}, \ldots, k_{m} \in\left\{k \in \mathbb{N}: 0 \leq k<2^{n}\right\}$ such that

$-r_{\xi_{i}} \in\left(\frac{k_{i}^{\prime}}{2^{n}}, \frac{k_{i}^{\prime}+1}{2^{n}}\right)$ for $1 \leq i \leq l$ and

$-r_{\xi_{i}^{\alpha}} \in\left(\frac{k_{i}}{2^{n}}, \frac{k_{i}+1}{2^{n}}\right)$ for $1 \leq i \leq m$;

- $f_{p_{\alpha}}^{\xi_{i}}=f_{i}^{\prime}$ for $1 \leq i \leq l$ and $f_{p_{\alpha}}^{\xi_{i}^{\alpha}}=f_{i}$ for $1 \leq i \leq m$ are rationally piecewise linear functions such that

$$
\begin{aligned}
& -f_{i}^{\prime}:\left[0, \frac{k_{i}^{\prime}}{2^{n}}\right] \cup\left[\frac{k_{i}^{\prime}+1}{2^{n}}, 1\right] \rightarrow[-1,1], \\
& -f_{i}:\left[0, \frac{k_{i}}{2^{n}}\right] \cup\left[\frac{k_{i}}{2^{n}}, 1\right] \rightarrow[-1,1] .
\end{aligned}
$$

Fix $\alpha<\beta<\omega_{1}$ as above. We will construct $o \leq p, q$ with $o \in \mathbb{P}$ where $p=p_{\alpha}$ and $q=p_{\beta}$. We will have $F_{o}=F_{p} \cup F_{q}$. Let $n_{o}>n$ be such a positive integer that there is an injective function sending $F_{o} \ni \xi \rightarrow k_{\xi}^{\prime \prime} \in\left\{k \in \mathbb{N}: 0 \leq k<2^{n_{o}}\right\}$ such that $r_{\xi} \in\left(\frac{k_{\xi}^{\prime \prime}}{2^{n_{o}}}, \frac{k_{\xi}^{\prime \prime}+1}{2^{n_{o}}}\right)$. It exists since all of our $r_{\xi} \mathrm{s}$ for $\xi<\omega_{1}$ are irrationals.

It is clear that we have

$$
\begin{aligned}
& \text { - } r_{\xi_{i}} \in\left(\frac{k_{\xi_{i}}^{\prime \prime}}{2^{n} o}, \frac{k_{\xi_{i}}^{\prime \prime}+1}{2^{n^{n}}}\right) \subseteq\left(\frac{k_{i}^{\prime}}{2^{n}}, \frac{k_{i}^{\prime}+1}{2^{n}}\right) \text { for } 1 \leq i \leq l \text { and } \\
& \text { - } r_{\xi_{i}^{\alpha}} \in\left(\frac{k_{\xi_{i}}^{\prime \prime}}{2^{\prime \prime}}, \frac{k_{\xi_{i}}^{\prime \prime}+1}{2^{n} n_{o}}\right) \subseteq\left(\frac{k_{i}}{2^{n}}, \frac{k_{i}+1}{2^{n}}\right) \text { for } 1 \leq i \leq m \text { and } \\
& \text { - } r_{\xi_{i}^{\beta}} \in\left(\frac{k_{i}^{\prime \prime}}{2^{n_{o}}}, \frac{k_{\xi_{i}}^{\prime \prime}+1}{2^{n_{o}}}\right) \subseteq\left(\frac{k_{i}}{2^{n}}, \frac{k_{i}+1}{2^{n}}\right) \text { for } 1 \leq i \leq m .
\end{aligned}
$$

So we are left with 
- extending each $f_{p}^{\xi_{i}^{\alpha}}$ for $\xi_{i}^{\alpha} \in F_{p} \backslash \Delta$ from $\left[0, \frac{k_{i}}{2^{n}}\right] \cup\left[\frac{k_{i}+1}{2^{n}}, 1\right]$ to a rationally piecewise linear function defined on $\left[0, \frac{k_{\xi_{i}^{\alpha}}^{\prime \prime}}{2^{n_{o}}}\right] \cup\left[\frac{k_{\xi_{i}^{\alpha}}^{\prime \prime}+1}{2^{n_{o}}}, 1\right]$ for $1 \leq i \leq m$,

- extending each $f_{q}^{\xi_{i}^{\beta}}$ for $\xi_{\beta}^{i} \in F_{q} \backslash \Delta$ from $\left[0, \frac{k_{i}}{2^{n}}\right] \cup\left[\frac{k_{i}+1}{2^{n}}, 1\right]$ to a rationally piecewise linear function defined on $\left[0, \frac{k_{i}^{\prime \prime}}{2^{n_{o}}}\right] \cup\left[\frac{k_{i}^{\prime \prime}+1}{2^{n_{o}}}, 1\right]$ for $1 \leq i \leq m$,

- extending each $f_{q}^{\xi_{i}}$ and $f_{p}^{\xi_{i}}$ for $\xi_{i} \in \Delta$ for $1 \leq i \leq l$ from $\left[0, \frac{k_{i}^{\prime}}{2^{n}}\right] \cup\left[\frac{k_{i}^{\prime}+1}{2^{n}}, 1\right]$ to a single (for each $1 \leq i \leq l$ ) rationally piecewise linear function defined on $\left[0, \frac{k_{\xi_{i}}^{\prime \prime}}{2^{n_{o}}}\right] \cup\left[\frac{k_{\xi_{i}}^{\prime \prime}+1}{2^{n_{o}}}, 1\right]$ for $1 \leq i \leq l$.

Obviously in the first two cases there are some extensions as above. In the third case we need to use the fact that for each $\xi_{i} \in \Delta$ both of the functions $f_{q}^{\xi_{i}}$ and $f_{p}^{\xi_{i}}$ are the same because they are equal to $f_{i}^{\prime}$ for $1 \leq i \leq l$. This completes the construction of $o \leq p, q$ and the proof of the c.c.c. for $\mathbb{P}$.

Proposition 4.4. $\mathbb{P}$ forces that there is an anti-Ramsey sequence $\left(f_{\xi}\right)_{\xi<\check{\omega}_{1}}$.

Proof. In the ground set-theoretic universe we fix a sequence $\left\{r_{\xi}: \xi<\omega_{1}\right\} \subseteq[0,1]$ of irrationals and force over this universe with $\mathbb{P}$ as in Definition 4.2 . In the generic extension for each $\alpha<\check{\omega}_{1}$ we choose finite pairwise disjoint $F_{\alpha}$ s and rationals $q_{i}, q_{i}^{\prime}$ for $1 \leq i \leq m \in \mathbb{N}$ and intervals $I_{i}$ s as in Definition 3.1 .

Let $\dot{G}$ be the standard $\mathbb{P}$-name for the generic filter in $\mathbb{P}$ (see [16]). In the generic extension define functions $f_{\xi}:[0,1] \backslash\left\{r_{\xi}\right\} \rightarrow[-1,1]$ as the unique continuous extension of $\bigcup\left\{f_{p}^{\xi}: p \in G\right\}$ to the new reals in $[0,1] \backslash\left\{r_{\xi}\right\}$. This can be done in a standard way since $f_{p}^{\xi}$ s are uniformly continuous in closed intervals away from $r_{\xi}$ respectively. The domain of $f_{\xi}$ is indeed $[0,1] \backslash\left\{r_{\xi}\right\}$ as using the technique of Lemma 4.3 one can check that

$$
\left\{p \in \mathbb{P}: n_{p}>n\right\}
$$

is dense in $\mathbb{P}$ for every $n \in \mathbb{N}$. From now on work in the ground universe. Let $\dot{f}_{\xi}$ be a $\mathbb{P}$-name for $f_{\xi}$. It is clear that each $p \in \mathbb{P}$ forces that $f_{p}^{\xi} \subseteq \dot{f}_{\xi}$. This will be sufficient for establishing the proposition. Fix $p \in \mathbb{P}$ and names $\dot{F}_{\alpha}$ such that $p$ forces that $\dot{F}_{\alpha} \mathrm{s}$ are pairwise disjoint $m$-element subsets of $\omega_{1}$. By going to a stronger condition we may assume that $p$ decides the endpoints of all intervals $I_{i}$ for $1 \leq i \leq m$. Let $d \in \mathbb{Q}$ be the diameter of the smallest of them. Let $p_{\alpha} \leq p$ and $F_{\alpha}=\left\{\xi_{1}^{\alpha}, \ldots, \xi_{m}^{\alpha}\right\}$ be in the ground universe such that $p_{\alpha} \Vdash \dot{F}_{\alpha}=\check{F}_{\alpha}$. We may assume that $F_{\alpha} \mathrm{s}$ form a $\Delta$-system, but since we have the c.c.c. and $p$ forces that they are pairwise disjoint the root of the $\Delta$-system must be empty, that is $F_{\alpha}$ s are pairwise disjoint. Noting that the sets

$$
\left\{p \in \mathbb{P}: F \subseteq F_{p}\right\}
$$

are dense in $\mathbb{P}$ for every finite $F \subseteq \omega_{1}$ by extending $p_{\alpha}$ s we may assume that $F_{\alpha} \subseteq F_{p_{\alpha}}$. As for every $\alpha<\omega_{1}$ the condition $p_{\alpha}$ forces that $r_{\xi_{i}^{\alpha}} \in I_{i}$ we may assume that there is a fixed $n^{\prime} \in \mathbb{N}$ such that $\left(r_{\xi_{i}^{\alpha}}-\frac{1}{2^{n^{\prime}}}, r_{\xi_{i}^{\alpha}}+\frac{1}{2^{n^{\prime}}}\right) \subseteq I_{i}$ for every $1 \leq i \leq m$. By going to an uncountable subsequence we may assume that the following holds for all $\alpha<\omega_{1}$ :

- $n_{p_{\alpha}}=n$ for some $n \in \mathbb{N}$ with $n \geq n^{\prime}$,

- $\left(F_{p_{\alpha}}\right)_{\alpha<\omega_{1}}$ forms a $\Delta$-system $F_{p_{\alpha}}=\Delta \cup\left\{\xi_{1}^{\alpha}, \ldots, \xi_{m}^{\alpha}, \xi_{m+1}^{\alpha} \ldots, \xi_{m^{\prime}}^{\alpha}\right\}$ with root $\Delta=\left\{\xi_{1}, \ldots, \xi_{l}\right\}$

- there are distinct $k_{1}^{\prime}, \ldots, k_{l}^{\prime}, k_{1}, \ldots, k_{m^{\prime}} \in\left\{k \in \mathbb{N}: 0 \leq k<2^{n}\right\}$ such that 
$-r_{\xi_{i}} \in\left(\frac{k_{i}^{\prime}}{2^{n}}, \frac{k_{i}^{\prime}+1}{2^{n}}\right)$ for $1 \leq i \leq l$ and

$-r_{\xi_{i}^{\alpha}} \in\left(\frac{k_{i}}{2^{n}}, \frac{k_{i}+1}{2^{n}}\right)$ for $1 \leq i \leq m^{\prime}$ and

$-\left(\frac{k_{i}}{2^{n}}, \frac{k_{i}+1}{2^{n}}\right) \subseteq I_{i}$ for $1 \leq i \leq m$ and

- $f_{p_{\alpha}}^{\xi_{i}}=f_{i}^{\prime}$ for $1 \leq i \leq l$ and $f_{p_{\alpha}}^{\xi_{i}^{\alpha}}=f_{i}$ for $1 \leq i \leq m^{\prime}$ and some rationally piecewise linear functions such that

$-f_{i}^{\prime}:\left[0, \frac{k_{i}^{\prime}}{2^{n}}\right] \cup\left[\frac{k_{i}^{\prime}+1}{2^{n}}, 1\right] \rightarrow[-1,1]$

$-f_{i}:\left[0, \frac{k_{i}}{2^{n}}\right] \cup\left[\frac{k_{i}+1}{2^{n}}, 1\right] \rightarrow[-1,1]$

Fix $\alpha<\beta<\omega_{1}$ as above. As in the proof of the c.c.c. of $\mathbb{P}$ we will construct $o \leq p, q$ where $p=p_{\alpha}$ and $q=p_{\beta}$ such that $o$ will force the properties $f_{\xi_{\alpha}^{i}}$ and $f_{\xi_{\beta}^{i}}$ as in Definition 3.1. We will have $F_{o}=F_{p} \cup F_{q}$. Let $n_{o}>n$ be such a positive integer that there is an injective function sending $F_{o} \ni \xi \rightarrow k_{\xi}^{\prime \prime} \in\left\{k \in \mathbb{N}: 0 \leq k<2^{n_{r}}\right\}$ such that $r_{\xi} \in\left(\frac{k_{\xi}^{\prime \prime}}{2^{n_{o}}}, \frac{k_{\xi}^{\prime \prime}+1}{2^{n_{o}}}\right)$. By choosing sufficiently big $n_{o}$ we may assume that

$\odot$

$$
\left|r_{\xi_{i}^{\alpha}}-\frac{k_{i}}{2^{n}}\right|,\left|r_{\xi_{i}^{\alpha}}-\frac{k_{i}+1}{2^{n}}\right|>\frac{1}{2^{n_{o}}}
$$

holds for every $1 \leq i \leq m$. It is clear that we have

- $r_{\xi_{i}} \in\left(\frac{k_{\xi_{i}}^{\prime \prime}}{2^{n_{o}}}, \frac{k_{\xi_{i}}^{\prime \prime}+1}{2^{n_{o}}}\right) \subseteq\left(\frac{k_{i}^{\prime}}{2^{n}}, \frac{k_{i}^{\prime}+1}{2^{n}}\right)$ for $1 \leq i \leq l$ and

- $r_{\xi_{i}^{\alpha}} \in\left(\frac{k_{\xi_{i}}^{\prime \prime}}{2^{n_{o}}}, \frac{k_{\xi_{i}}^{\prime \prime}+1}{2^{n_{o}}}\right) \subseteq\left(\frac{k_{i}}{2^{n}}, \frac{k_{i}+1}{2^{n}}\right)$ for $1 \leq i \leq m^{\prime}$ and

- $r_{\xi_{i}^{\beta}} \in\left(\frac{k_{i}^{\prime \prime}}{2^{n_{o}}}, \frac{k_{\xi_{i}}^{\prime \prime}+1}{2^{n_{o}}}\right) \subseteq\left(\frac{k_{i}}{2^{n}}, \frac{k_{i}+1}{2^{n}}\right)$ for $1 \leq i \leq m^{\prime}$.

We define

- $J_{i}^{\alpha}=\left(\frac{k_{\xi_{i}}^{\prime \prime}}{2^{n_{o}}} \frac{k_{\xi_{i}}^{\prime \prime}+1}{2^{n_{o}}}\right)$ for $1 \leq i \leq m$,

- $J_{i}^{\beta}=\left(\frac{k_{i}^{\prime \prime}}{2^{n_{o}}}, \frac{k_{\xi_{i}^{\prime}}^{\prime \prime}+1}{2^{n_{o}}}\right)$ for $1 \leq i \leq m$.

So we have $J_{i}^{\alpha}, J_{i}^{\beta} \subseteq I_{i}$ for each $1 \leq i \leq m$ but by $\odot$ the endpoints of $J_{i}$ s do not coincide with the endpoints of the intervals $\left[\frac{k}{2^{n}}, \frac{k+1}{2^{n}}\right]$ for $0 \leq k<2^{n}$. We are left with

- extending each $f_{p}^{\xi_{i}^{\alpha}}$ for $\xi_{i}^{\alpha} \in F_{p} \backslash \Delta$ from $\left[0, \frac{k_{i}}{2^{n}}\right] \cup\left[\frac{k_{i}+1}{2^{n}}, 1\right]$ to a rationally piecewise linear function $f_{o}^{\xi_{i}^{\alpha}}$ defined on

$$
\left[0, \frac{k_{\xi_{i}^{\alpha}}^{\prime \prime}}{2^{n_{o}}}\right] \cup\left[\frac{k_{\xi_{i}^{\alpha}}^{\prime \prime}+1}{2^{n_{o}}}, 1\right]=[0,1] \backslash J_{i}^{\alpha},
$$

and extending each $f_{q}^{\xi_{i}^{\beta}}$ for $\xi_{i}^{\beta} \in F_{q} \backslash \Delta$ from $\left[0, \frac{k_{i}}{2^{n}}\right] \cup\left[\frac{k_{i}+1}{2^{n}}, 1\right]$ to a rationally piecewise linear function $f_{o}^{\xi_{i}^{\beta}}$ defined on

$$
\left[0, \frac{k_{\xi_{i}^{\beta}}^{\prime \prime}}{2^{n_{o}}}\right] \cup\left[\frac{k_{\xi_{i}^{\beta}}^{\prime \prime}+1}{2^{n_{o}}}, 1\right]=[0,1] \backslash J_{i}^{\beta},
$$

so that

(1) $f_{o}^{\xi_{i}^{\alpha}} \uparrow\left([0,1] \backslash\left(J_{i}^{\alpha} \cup J_{i}^{\beta}\right)\right)=f_{o}^{\xi_{i}^{\beta}} \uparrow\left([0,1] \backslash\left(J_{i}^{\alpha} \cup J_{i}^{\beta}\right)\right)$,

(2) $f_{o}^{\xi_{i}^{\alpha}} \uparrow J_{i}^{\beta}=q_{i}$,

(3) $f_{o}^{\xi_{i}^{\beta}} \uparrow J_{i}^{\alpha}=q_{i}^{\prime}$.

- extending each $f_{q}^{\xi}$ and $f_{p}^{\xi}$ for $\xi \in \Delta$ from $\left[0, \frac{k_{i}^{\prime}}{2^{n}}\right] \cup\left[\frac{k_{i}^{\prime}+1}{2^{n}}, 1\right]$ to a single for each $\xi \in \Delta$ rationally piecewise linear function defined on $\left[0, \frac{k_{\xi}^{\prime \prime}}{2^{n_{o}}}\right] \cup\left[\frac{k_{\xi}^{\prime \prime}+1}{2^{n_{o}}}, 1\right]$, 
As we noted in the proof of the c.c.c. of $\mathbb{P}$ any extensions will do to obtain $o \leq p, q$, however to obtain the properties of $f_{\xi_{\alpha}^{i}}$ and $f_{\xi_{i}^{\beta}}$ from Definition 3.1 one needs to follow the requirements (3) - (5) of it that is obtain (1)-(3) above. This can be achieved as $f_{p}^{\xi_{i}^{\alpha}}=f_{q}^{\xi_{i}^{\beta}}=f_{i}$ which is defined on $[0,1] \backslash\left(\frac{k_{i}}{2^{n}}, \frac{k_{i}+1}{2^{n}}\right)$ and $J_{i}^{\alpha}, J_{i}^{\beta} \subseteq$ $\left[\frac{k_{i}}{2^{n}}, \frac{k_{i}+1}{2^{n}}\right]$ are disjoint for each $1 \leq i \leq m$ and have the endpoints distinct from $\frac{k_{i}}{2^{n}}, \frac{k_{i}+1}{2^{n}}$ by $\odot$.

In the third case we need to use the fact that for each $\xi_{i} \in \Delta$ both of the functions $f_{q}^{\xi_{i}}$ and $f_{p}^{\xi_{i}}$ are the same because they are equal to $f_{i}^{\prime}$ for $1 \leq i \leq l$.

This completes the construction of $o \leq p, q$ which forces the properties $f_{\xi_{i}^{\alpha}}$ and $f_{\xi_{i}^{\beta}}$ from Definition 3.1 .

Theorem 4.5. It is relatively consistent with $Z F C$ that there is a sequence $\left(f_{\xi}\right)_{\xi<\omega_{1}}$ which is anti-Ramsey.

Proof. As $\mathbb{P}$ is c.c.c. by Lemma 4.3 the $\omega_{1}$ of the generic extension is the same as $\omega_{1}$ of the ground universe, and so Proposition 4.4 implies that there is an anti-Ramsey sequence in the generic extension.

\section{Equilateral sets in Banach spaces of the form $C(K)$ under MARTIN'S AXIOM}

Theorem 5.1. Assume Martin's axiom and the negation of the continuum hypothesis. Suppose that $K$ is a compact Hausdorff space. If the Banach space $C(K)$ of real valued continuous functions on $K$ wit the supremum norm is nonseparable, then the unit ball of $C(K)$ contains

- an uncountable 2-equilateral set,

- an uncountable $(1+\varepsilon)$-separated set.

Proof. First let us note that we may assume that the weight of $K$, that is the density of $C(K)$ is the first uncountable cardinal $\omega_{1}$. Indeed, by embedding $K$ into $[0,1]^{\kappa}$ for some uncountable $\kappa$ one may find an uncountable $A \subseteq \kappa$ such that its cardinality is $\omega_{1}$ and the projection from $[0,1]^{\kappa}$ onto $[0,1]^{A}$ sends $K$ to a set of uncountable weight. So we obtain a continuous image $L$ of $K$ which is of weight $\omega_{1}$, it is clear that $C(L)$ has its isometric copy inside $C(K)$ and so an equilateral set in $C(L)$ yields an equilateral set in $C(K)$. We may also assume that $K$ is hereditarily Lindelöf by Theorems 1 and 2 (2) of [19]. We will need the following fact about hereditarily Lindelöf compact Hausdorff spaces:

Claim: If $K$ is compact Hausdorff hereditarily Lindelöf, $U, V$ are disjoint open subsets of $K$, then there is a continuous function $f: K \rightarrow[-1,1]$ such that $f(x)>0$ for all $x \in U$ and $f(x)<0$ for all $x \in V$.

Proof of the Claim: By the regularity of $K$ both $U$ and $V$ can be covered by their open subsets whose closures are included in $U$ and $V$ respectively. As $K$ is hereditarily Lindelöf, we can find countable subcovers of these covers which yield sequences of open sets $\left(U_{n}\right)_{n \in \mathbb{N}}$ and $\left(V_{n}\right)_{n \in \mathbb{N}}$ such that $\bigcup_{n \in \mathbb{N}} U_{n}=U, \bigcup_{n \in \mathbb{N}} V_{n}=V$ and $\overline{U_{n}} \subseteq U$ as well as $\overline{V_{n}} \subseteq V$ hold for each $n \in \mathbb{N}$. It follows that $\overline{U_{n}} \cap \bar{V}=\emptyset$ as well as $\overline{V_{n}} \cap \bar{U}=\emptyset$ for each $n \in \mathbb{N}$. Using the normality of $K$ define $f_{n}: K \rightarrow\left[0, \frac{1}{2^{n}}\right]$ such that $f_{n} \uparrow \overline{U_{n}}=\frac{1}{2^{n}}$ and $f_{n} \uparrow \bar{V}=0$ and likewise $g_{n}: K \rightarrow\left[\frac{-1}{2^{n}}, 0\right]$ such that $g_{n}\left\lceil\overline{V_{n}}=\frac{-1}{2^{n}}\right.$ and $g_{n}\left\lceil\bar{U}=0\right.$. Now $f=\Sigma_{n \in \mathbb{N}}\left(f_{n}+g_{n}\right)$ works as $\bigcup_{n \in N} \overline{U_{n}}=U$ and $\bigcup_{n \in N} \overline{V_{n}}=V$ which completes the proof of the claim. 
Let $\mathcal{B}$ be an open basis for $K$ of size $\omega_{1}$. Consider the partial ordering $\leq$ on the set

$$
\mathbb{P}=\left\{p=\left(U_{p}, V_{p}\right): \overline{U_{p}} \cap \overline{V_{p}}=\emptyset ; U_{p}, V_{p} \in \mathcal{B}\right\}
$$

where $p \leq q$ if and only if $U_{p} \supseteq U_{q}$ and $V_{p} \supseteq V_{q}$. Let us assume that $\mathbb{P}$ is c.c.c. and we will derive a contradiction with the nonmetrizability of $K$. By the negation of the continuum hypothesis $|\mathbb{P}|<2^{\omega}$ and so by Martin's axiom and Theorem 4.5 from [30] $\mathbb{P}=\bigcup_{n \in \mathbb{N}} \mathbb{P}_{n}$ where each $\mathbb{P}_{n}$ consists of compatible elements. That is, for each $n \in \mathbb{N}$ and each $p, q \in \mathbb{P}_{n}$ there is $r \in \mathbb{P}$ such that $r \leq p, q$ which in particular means that $\overline{\left(U_{p} \cup U_{q}\right)} \cap \overline{\left(V_{p} \cup V_{q}\right)}=\emptyset$. Consider $\bigcup\left\{U_{p}: p \in \mathbb{P}_{n}\right\}=U_{n}$ and $\bigcup\left\{V_{p}: p \in \mathbb{P}_{n}\right\}=V_{n}$ for each $n \in \mathbb{N}$ and note that the compatibility of the elements of $\mathbb{P}_{n}$ gives that $U_{n} \cap V_{n}=\emptyset$ for each $n \in \mathbb{N}$. Hence, following the claim, we can construct continuous $f_{n}: K \rightarrow[-1,1]$ such that $f \nmid U_{n}$ is strictly positive and $f \nmid V_{n}$ is strictly negative. Now we note that given two distinct points $x, y \in K$ there is $p \in \mathbb{P}$ such that $x \in U_{p}$ and $y \in V_{p}$ as the open sets are taken from a basis and hence there is an $n \in \mathbb{N}$ such that $f_{n}$ separates $x$ and $y$. The existence of a countable family of continuous functions separating the points of $K$ contradicts the nonmetrizability of $K$.

So we conclude that $\mathbb{P}$ has an uncountable antichain $\left(p_{\xi}: \xi<\omega_{1}\right)$. It means that $\overline{U_{p_{\xi}}} \cap \overline{V_{p_{\eta}}} \neq \emptyset$ or $\overline{V_{p_{\xi}}} \cap \overline{U_{p_{\eta}}} \neq \emptyset$ for any distinct $\xi, \eta<\omega_{1}$ and in particular $\left(U_{p_{\xi}}, V_{p_{\eta}}\right) \neq\left(U_{p_{\eta}}, V_{p_{\xi}}\right)$, hence we obtain an uncountable intersecting family of closed pairs which is equivalent by Theorem 1 of [19] to the existence of 2-equilateral uncountable family in the sphere of $C(K)$ and an $(1+\varepsilon)$-separated set for some $\varepsilon>0$.

\section{REFERENCES}

1. S. Argyros, J. Lopez-Abad, S. Todorcevic, A class of Banach spaces with few non-strictly singular operators. J. Funct. Anal. 222 (2005), no. 2, 306-384.

2. A. Avilés, P. Koszmider, A continuous image of a Radon-Nikodym compact space which is not Radon-Nikodym. Duke Math. J. 162 (2013), no. 12, 2285-2299.

3. J. Baumgartner, O. Spinas, Independence and consistency proofs in quadratic form theory. J. Symbolic Logic 56 (1991), no. 4, 1195-1211.

4. C. Brech, P. Koszmider, On biorthogonal systems whose functionals are finitely supported. Fund. Math. 213 (2011), no. 1, 43-66.

5. K. Ciesielski, Set theory for the working mathematician. London Mathematical Society Student Texts, 39. Cambridge University Press, Cambridge, 1997.

6. J. Elton, E. Odell, The unit ball of every infinite-dimensional normed linear space contains $a(1+\varepsilon)$-separated sequence. Colloq. Math. 44 (1981), no. 1, 105-109.

7. M. Fabian, P. Habala, P. Hajek, V. Montesinos Santalucia, J. Pelant, V. Zizler, Functional analysis and infinite-dimensional geometry. CMS Books in Mathematics/Ouvrages de Mathématiques de la SMC, 8. Springer-Verlag, New York, 2001.

8. C. Finet, G. Godefroy, Biorthogonal systems and big quotient spaces. Banach space theory (Iowa City, IA, 1987), 87-110, Contemp. Math., 85, Amer. Math. Soc., Providence, RI, 1989.

9. E. Glakousakis, S. Mercourakis Examples of infinite dimensional Banach spaces without infinite equilateral sets. Preprint: arxiv.org/abs/1502.02500

10. T. Kania, T. Kochanek, Uncountable sets of unit vectors that are separated by more than 1 . Preprint: arxiv.org/abs/1503.08166

11. P. Koszmider, Forcing minimal extensions of Boolean algebras. Trans. Amer. Math. Soc. 351 (1999), no. 8, 3073-3117.

12. P. Koszmider, Banach spaces of continuous functions with few operators. Math. Ann. 330 (2004), no. 1, 151-183.

13. P. Koszmider, The interplay between compact spaces and the Banach spaces of their continuous functions, in Open Problems in Topology 2; ed. Elliott Pearl, Elsevier 2007. 
14. P. Koszmider, On a problem of Rolewicz about Banach spaces that admit support sets. J. Funct. Anal. 257 (2009), no. 9, 2723-2741.

15. C. Kottman, Subsets of the unit ball that are separated by more than one. Studia Math. 53 (1975), no. 1, 15-27.

16. K. Kunen, Set theory. An introduction to independence proofs. Studies in Logic and the Foundations of Mathematics, 102. North-Holland Publishing Co., Amsterdam-New York, 1980.

17. J. Lopez-Abad, S. Todorcevic, A co-saturated Banach space with no long unconditional basic sequences. Trans. Amer. Math. Soc. 361 (2009), no. 9, 4541-4560

18. S. Mercourakis, G. Vassiliadis, Equilateral sets in infinite dimensional Banach spaces. Proc. Amer. Math. Soc. 142 (2014), no. 1, 205-212.

19. S. Mercourakis, G. Vassiliadis, Equilateral Sets in Banach Spaces of th form $C(K)$ Preprint: http://arxiv.org/abs/1501.06381

20. I. Schlackow, Centripetal operators and Koszmider spaces. Topology Appl. 155 (2008), no. 11, $1227-1236$.

21. S. Shelah, On uncountable Boolean algebras with no uncountable pairwise comparable or incomparable sets of elements. Notre Dame J. Formal Logic 22 (1981), no. 4, 301-308.

22. S. Shelah, Uncountable constructions for B.A., e.c. groups and Banach spaces. Israel J. Math. 51 (1985), no. 4, 273-297.

23. S. Shelah, J. Steprans, A Banach space on which there are few operators. Proc. Amer. Math. Soc. 104 (1988), no. 1, 101-105.

24. P. Terenzi, Equilater sets in Banach spaces. Boll. Un. Mat. Ital. A (7) 3 (1989), no. 1, 119-124.

25. S. Todorcevic, Partitioning pairs of countable ordinals. Acta Math. 159 (1987), no. 3-4, 261294.

26. S. Todorcevic, Biorthogonal systems and quotient spaces via Baire category methods, Math. Ann. 335 (2006), no. 3, 687-715.

27. S. Todorcevic, Walks on ordinals and their characteristics. Progress in Mathematics, 263. Birkhauser Verlag, Basel, 2007.

28. H. Wark, A non-separable reflexive Banach space on which there are few operators. J. London Math. Soc. (2) 64 (2001), no. 3, 675-689.

29. N. Weaver, Forcing for mathematicians. World Scientific Publishing Co. Pte. Ltd., Hackensack, NJ, 2014.

30. W. A. R. Weiss, Versions of Martin's axiom. Handbook of set-theoretic topology, 827-886, North-Holland, Amsterdam, 1984.

Institute of Mathematics, Polish Academy of Sciences, ul. Śniadeckich 8, 00-656 WARSZAWA, POLAND

E-mail address: piotr.koszmider@impan.pl 